Case Report

\title{
A Unique Case of Primary Ewing's Sarcoma of the Cervical Spine in a 53-Year-Old Male: A Case Report and Review of the Literature
}

\author{
Marshall T. Holland, Oliver E. Flouty, Liesl N. Close, \\ Chandan G. Reddy, and Matthew A. Howard III \\ Department of Neurosurgery, University of Iowa, 200 Hawkins Drive, Iowa City, IA 52246, USA \\ Correspondence should be addressed to Marshall T. Holland; marshall-holland@uiowa.edu
}

Received 28 December 2014; Accepted 10 February 2015

Academic Editor: Thomas R. Chauncey

Copyright (C) 2015 Marshall T. Holland et al. This is an open access article distributed under the Creative Commons Attribution License, which permits unrestricted use, distribution, and reproduction in any medium, provided the original work is properly cited.

\begin{abstract}
Extraskeletal Ewing's sarcoma (EES) is a rare presentation, representing only $15 \%$ of all primary Ewing's sarcoma cases. Even more uncommon is EES presenting as a primary focus in the spinal canal. These rapidly growing tumors often present with focal neurological symptoms of myelopathy or radiculopathy. There are no classic characteristic imaging findings and thus the physician must keep a high index of clinical suspicion. Diagnosis can only be definitively made by histopathological studies. In this report, we discuss a primary cervical spine EES in a 53-year-old man who presented with a two-month history of left upper extremity pain and acute onset of weakness. Imaging revealed a cervical spinal canal mass. After undergoing cervical decompression, histopathological examination confirmed a diagnosis of Ewing's sarcoma. A literature search revealed fewer than 25 reported cases of primary cervical spine EES published in the past 15 years and only one report demonstrating this pathology in a patient older than 30 years of age $($ age $=38)$. Given the low incidence of this pathology presenting in this age group and the lack of treatment guidelines, each patient's plan should be considered on a case-by-case basis until further studies are performed to determine optimal evidence based treatment.
\end{abstract}

\section{Introduction}

Ewing's sarcoma is a malignant primitive neuroectodermal tumor (PNET) that primarily presents during the first two decades of life. Approximately $85 \%$ of those cases present primarily in the skeleton. With an annual incidence of 1-3 per million, it is generally a rare diagnosis [1]. EES occurs most often during the second or third decade of life. While the spinal canal remains a rare site for Ewing's sarcoma to arise, more common sites for an EES to present include the head and neck, buttocks, lower extremities, chest wall, and retroperitoneal space [2]. There have been examples of both intradural and extradural presentations of primary spinal EES $[3,4]$. Primary spinal EES has a predilection for the sacral spine, with $50 \%$ of spinal EES appearing in this location [5]. Although reported, it is unusual to see cervical spine involvement in a primary EES $[5,6]$. Acute to subacute localizable pain, myelopathic symptoms, and radicular symptoms are common presenting complaints. Upon performing a PubMed Search for English language articles on EES, we found less than 25 reported cases of EES with at least partial involvement of the cervical spine in the past 15 years. All of these have occurred in patients less than 40 years of age [5, 7-21].

\section{Case Report}

2.1. History and Physical Examination. A 53-year-old male first presented to his primary care provider with a 2-month history of progressive complaints that started as a stiff neck and subjective right shoulder blade mass that was never objectively noted by any physician with some right upper extremity pain. This pain reportedly then spread to the left upper extremity with pain radiating down the posterior side to the left proximal wrist. Due to these complaints, he was evaluated by his primary care provider and started on ibuprofen. Upon awakening the following morning, the patient 


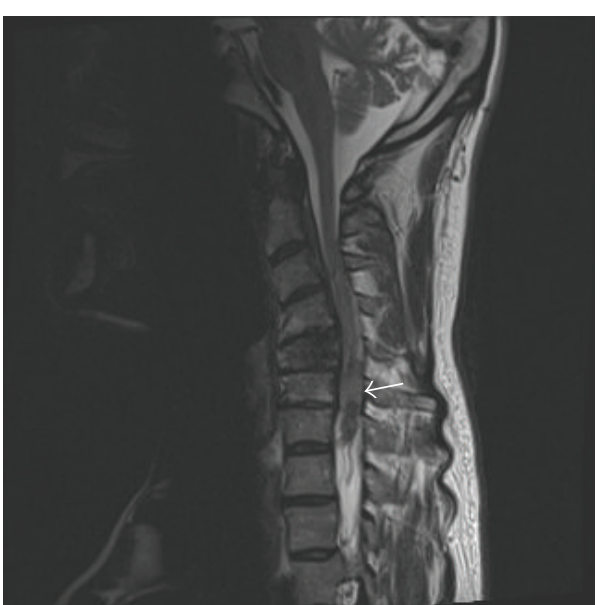

(a)

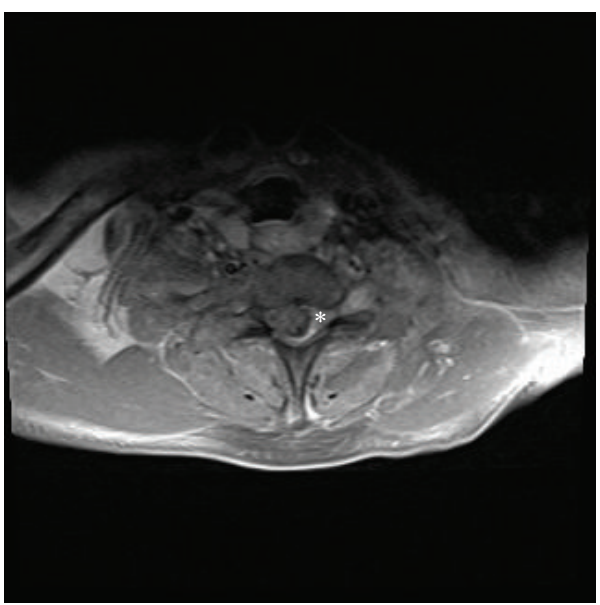

(c)

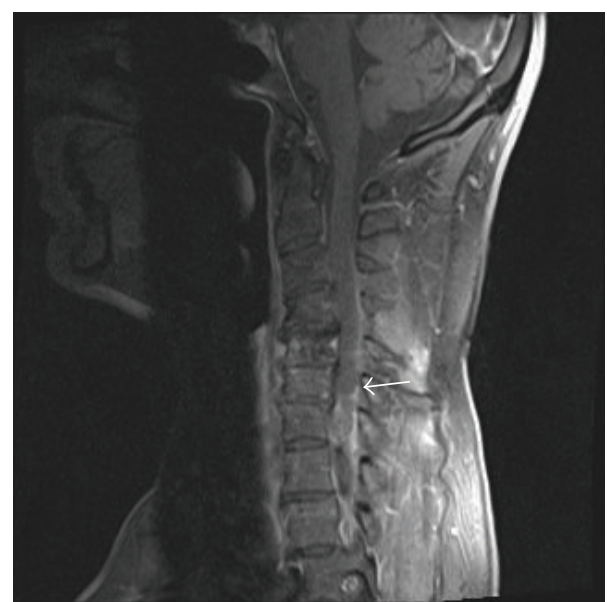

(b)

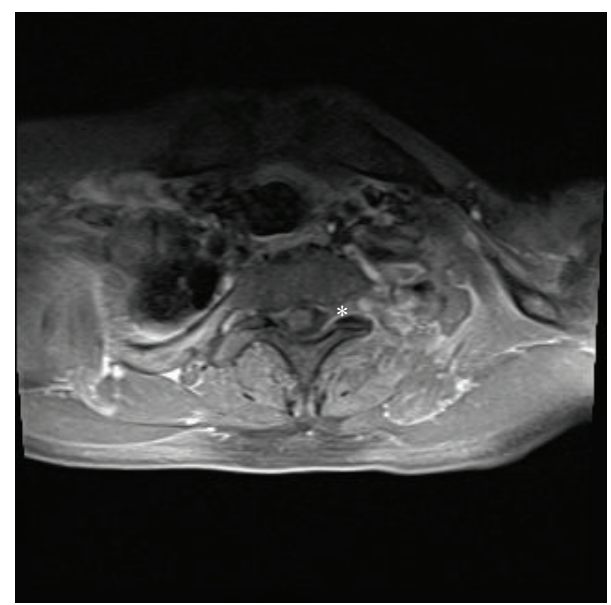

(d)

Figure 1: Cervical MRI: (a) midsagittal T2 MRI $(\mathrm{TR}=4000, \mathrm{TE}=98)$ and $(\mathrm{b})$ midsagittal $\mathrm{T} 1$ postcontrast $(\mathrm{TR}=659, \mathrm{TE}=8.7)$ imaging of the cervical spine showing a C2-C7 ill-defined extradural mass that is hypointense on T2 and enhances with contrast most predominant at C6-C7. ((c) and (d)) Two axial slices of a preoperative T1 postgadolinium contrast $(\mathrm{TR}=591$, TE $=7.3)$ MRI of the cervical spine passing through the C6-C7 level demonstrates an extradural, enhancing mass invading the spinal canal eccentric to the left.

reported the sudden onset of left arm weakness that was not antigravity. Conversely however, the left upper extremity pain had remitted. With this sudden change in neurological function, the patient's primary care provider obtained an MRI of the cervical spine. The patient was told that the MRI showed a bony lesion and he was referred to our institution's orthopedics clinic. One month later he presented to the orthopedic clinic where the patient noted that his left upper extremity pain had returned. The patient and his wife also noted that, over the previous two-month period, he was losing muscle mass most profoundly in the left biceps and deltoid. Furthermore, he had also unintentionally lost 10 pounds over the previous three months. Due to his suspicious presentation and cervical spine lesion noted on the MRI, the patient was referred to our neurosurgical clinic.

On examination, the patient appeared emaciated and easily fatigable. Muscle strength testing revealed 2/5 in the left upper extremity with exception of the biceps at $3 / 5$ and handgrip of $4-/ 5$. His right upper extremity was $3 / 5$ in the deltoid and 4-/5 in all other muscle groups. The bilateral lower extremities were 5/5 for strength. There were no myelopathic signs. A repeat MRI performed at our institution showed an ill-defined predominantly extradural enhancement along the left aspect of the cervical spinal canal, most apparent at C6C7, but also extending between C2 and C7 (Figure 1). A CT chest/abdomen/pelvis showed no evidence of other primary diseases. After being admitted to the hospital, the patient acutely developed increased difficulty with his gait and experienced an episode of urinary incontinence.

2.2. Operation. The patient underwent a C6-C7 laminotomy with decompression of the left C7 nerve root and biopsy of the extradural lesion, accomplishing the goals of operation including decompression and definitive diagnosis. Intraoperative baseline SSEPs were attempted; however no electrophysiological recordings could be obtained apart from minimal recordings in the right upper extremity. 


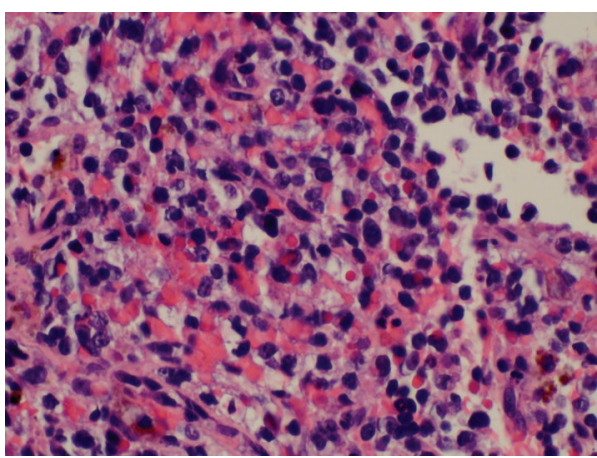

(a)

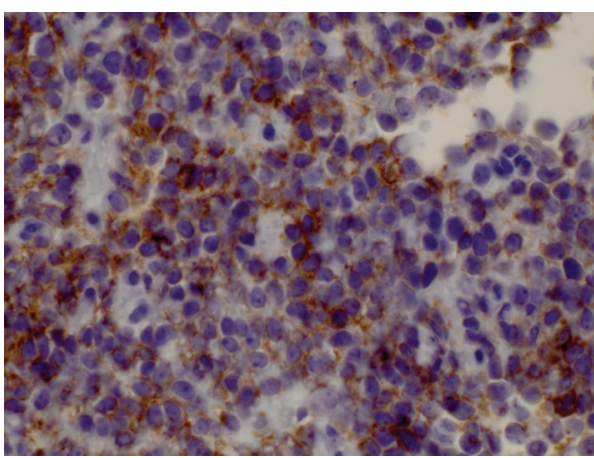

(c)

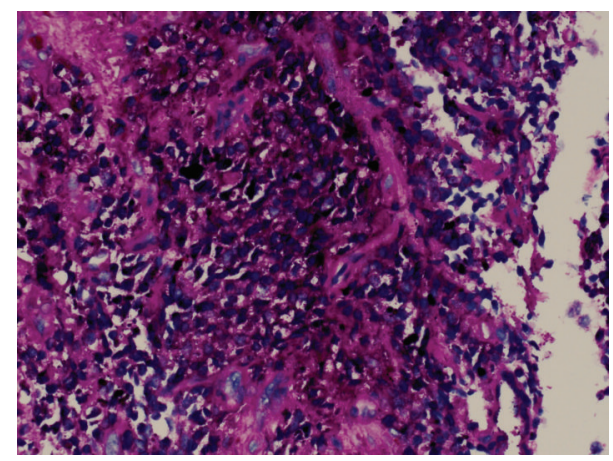

(b)

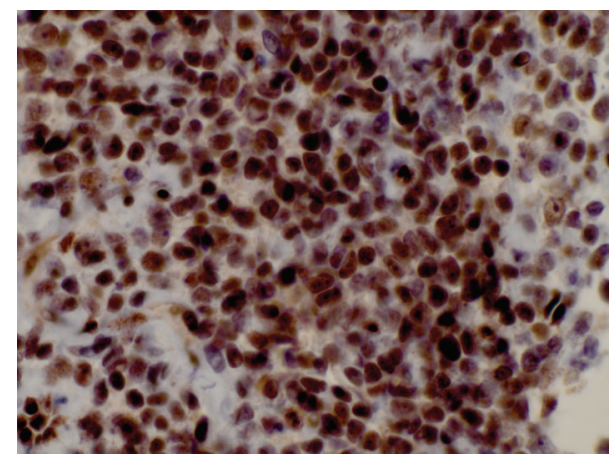

(d)

FIGURE 2: Histopathological investigation of the biopsied mass included (a) H\&E staining that revealed round hyperchromatic nuclei with little cytoplasm and numerous mitoses. (b) Positive PAS staining of the tumor cells shows these cells to be glycogen rich. There is also positive staining for (c) CD-99 and (d) FLI-1.

2.3. Pathological Findings. Histopathological studies established the diagnosis of Ewing's sarcoma. Our pathologists noted round, hyperchromatic nuclei with little cytoplasm and multiple mitosis suggestive of a primitive neuroectodermal tumor in surrounding necrotic tissue (Figure 2(a)). Further histochemical staining showed that the tumor cells stained positively for PAS, CD-99 and FLI-1 (Figures 2(c) and 2(d)). The cells stained negative for pankeratin, S100, synatophysin, chromogranin CD45, CD5, and CD20. Following these immunohistochemical protocols, the diagnosis of Ewing's sarcoma was confirmed.

2.4. Postoperative Course. Upon awakening postoperatively, the patient was noted to be severally quadriparetic and had suffered a myocardial infarction. He was transferred to the surgical intensive care unit where he was kept on flat bedrest and high mean arterial pressure control. He recovered remarkably well. First, he regained movement in his lower extremities and subsequently regained antigravity ability in his right upper extremity. He continued to improve with the help of physical and occupational therapy. At his 6-month follow-up appointment his muscle strength was slowly improving with $3 / 5$ in the left upper extremity with exception of handgrip, which was $4-/ 5$. His right upper extremity was $4-/ 5$ throughout. The bilateral lower extremities were $4 / 5$ in strength. He was started on a course of chemotherapy and radiation therapy prior to discharge. To date, he has completed radiation therapy with a total treatment of $55.8 \mathrm{~Gy}$. $\mathrm{He}$ is currently undergoing infusion treatment sessions of vincristine, actinomycin, and cyclophosphamide alternating with ifosfamide and etoposide.

\section{Discussion}

Extraskeletal Ewing's sarcoma of the spinal canal remains a rare diagnosis. It has been a subject of interest since its first published description in 1969 [22]. EES affects males as often as females during the second or third decade of life, slightly later than the classic age of presentation for primary skeletal Ewing's sarcoma $[8,23]$. The case presented here is a rare example of a primary extraskeletal Ewing's sarcoma of the cervical spinal canal. Furthermore, this case occurred in an adult patient in his sixth decade of life, far outside the typical presenting age range, 15 years older than any previously reported case. Ewing's sarcoma classically presents with local swelling and pain from the rapidly enlarging mass. When this occurs in the spinal canal, this can, as in our patient, initially present with pain and progress to other neurological symptoms such as weakness, numbness, tingling, or gait disturbances [5, $7,8,18,24]$. It is important to emphasize that these signs and symptoms can progress fairly rapidly, as in our case, warranting urgent surgical intervention [25].

In reviewing previously published case reports of primary EES of the cervical spine there were some patterns of 
TABLE 1: Summary review: primary EES of cervical spine (available in English).

\begin{tabular}{|c|c|c|c|c|c|}
\hline Authors & $\begin{array}{c}\text { Number of } \\
\text { cases }\end{array}$ & $\begin{array}{c}\text { Year of } \\
\text { publishing }\end{array}$ & $\begin{array}{c}\text { Cervical } \\
\text { level }\end{array}$ & Age/gender & Presenting symptoms \\
\hline Martin Garcia et al. [14] & 1 & 1991 & $\mathrm{C} 2$ & $15 / \mathrm{M}$ & Right posterior neck pain \\
\hline Sharafuddin et al. [17] & 1 & 1992 & C5 & $21 / \mathrm{F}$ & $\begin{array}{l}\text { Neck pain and RUE radicular pain, progressive spastic } \\
\text { quadriparesis }\end{array}$ \\
\hline Villas and San Julian [19] & 1 & 1996 & C6 & $14 /(\mathrm{n} / \mathrm{a})$ & $\mathrm{n} / \mathrm{a}$ \\
\hline Kennedy et al. [8] & 1 & 2000 & $\mathrm{C} 1-\mathrm{C} 5$ & $24 / \mathrm{M}$ & Neck pain and neck mass \\
\hline \multirow{2}{*}{$\begin{array}{l}\text { Mukhopadhyay et al. } \\
\text { [15] }\end{array}$} & \multirow{2}{*}{2} & \multirow{2}{*}{2001} & $\mathrm{C} 3-\mathrm{C} 5$ & $29 / \mathrm{F}$ & Acute progressive quadriparesis and urinary retention \\
\hline & & & $\mathrm{C} 3-\mathrm{C} 5$ & $13 / \mathrm{M}$ & Severe back pain \\
\hline \multirow{2}{*}{ Shin et al. [18] } & \multirow{2}{*}{2} & \multirow{2}{*}{2001} & $\mathrm{C} 7-\mathrm{T} 1$ & $22 / \mathrm{F}$ & Right arm paresthesia \\
\hline & & & $\mathrm{C} 5-\mathrm{C} 7$ & $38 / \mathrm{M}$ & Right shoulder pain \\
\hline Venkateswaran et al. [28] & 2 & 2001 & $\mathrm{n} / \mathrm{a}$ & $\mathrm{n} / \mathrm{a}$ & Local pain \\
\hline Ilaslan et al. [5] & 4 & 2004 & $\mathrm{n} / \mathrm{a}$ & $\mathrm{n} / \mathrm{a}$ & Local pain \\
\hline Kara [12] & 1 & 2004 & C5-C6 & $18 / \mathrm{F}$ & Back pain and fever \\
\hline Kogawa et al. [13] & 1 & 2004 & $\mathrm{C} 2-\mathrm{C} 4$ & $7 / \mathrm{F}$ & Neck pain and LUE weakness \\
\hline Bozkurt et al. [7] & 1 & 2007 & $\mathrm{C} 2-\mathrm{C} 5$ & $28 / \mathrm{M}$ & Neck pain and gait disturbance \\
\hline Ozturk et al. [16] & 1 & 2007 & C6-T1 & $18 / \mathrm{M}$ & $\begin{array}{l}\text { Right neck and shoulder pain with progression to } \\
\text { paraplegia and urinary retention }\end{array}$ \\
\hline Ali et al. [9] & 1 & 2008 & $\mathrm{C} 6-\mathrm{T} 1$ & $14 / \mathrm{M}$ & Neck mass \\
\hline Bacci et al. [29] & 2 & 2009 & $\mathrm{n} / \mathrm{a}$ & $\mathrm{n} / \mathrm{a}$ & $\mathrm{n} / \mathrm{a}$ \\
\hline Hao et al. [11] & 1 & 2010 & C6 & $15 / \mathrm{M}$ & Neck/shoulder pain RUE numbness \\
\hline Gulati et al. [21] & 1 & 2011 & $\mathrm{C} 2$ & $11 / \mathrm{F}$ & Nonradiating neck pain \\
\hline Ellis et al. [31] & 1 & 2011 & $\mathrm{C} 5-\mathrm{C} 7$ & $27 / \mathrm{M}$ & Acute ascending weakness and sensory changes \\
\hline Cabral et al. [10] & 1 & 2012 & $\mathrm{C} 1-\mathrm{C} 2$ & $22 / \mathrm{F}$ & Neck pain and right arm paresthesia \\
\hline \multirow[t]{2}{*}{ Current case } & 1 & 2013 & $\mathrm{C} 1-\mathrm{C} 7$ & $53 / \mathrm{M}$ & LUE pain and weakness \\
\hline & 26 & & & & \\
\hline
\end{tabular}

n/a: not available.

presentation. The average age at presentation was 19.7 years and there were no cases identified that presented in a patient past 38 years of age. There was a slight propensity for the pathology to present in males $(42 \%)$ compared to females $(29 \%)$ in cases where gender was specified. However, one must take into account that $29 \%$ of the cases identified did not report the patient's gender. A further breakdown of these previous cases is summarized in Table 1.

Due to the rarity of this pathology, there are few large retrospective studies available for review. However, one such study performed by Ilaslan at the Mayo Clinic was able to identify 125 patients with primary spinal EES out of 1,277 patients with primary Ewing's sarcoma treated at the clinic from 1926 to 2001. The average age of the patients was 19.3 years (range 4-54 years). When the EES was organized by level in the spinal column, there were only a total of 4 patients identified (3\%), further showing the infrequency of this pathology [5].

In terms of imaging, reports vary and there appears to be no classical findings of an EES mass. On CT, there tends to be a mass that enhances heterogeneously [18]. On MRI imaging the mass may appear hypo-, iso-, or hyperintense on $\mathrm{T} 1$ and hypo or hyperintense on T2. The mass often appears to enhance heterogeneously $[8,26]$. Thus PNET should be included in the differential diagnosis of nonspecific spinal canal lesions. Our lesion was hypodense on T1 and slightly hyperintense on $\mathrm{T} 2$ and enhanced with contrast administration (Figure 1).

Definitive diagnosis is made by histopathological studies. Findings include uniform small round blue cells with scant possibly slightly eosinophilic cytoplasm notable on light microscopy. Two cellular markers frequently employed by pathologists are CD-99, which is expressed in both Ewing's sarcoma and lymphoma, and CD-45, which is only expressed in lymphoblastic lymphoma [1]. Typically, in order to confirm a diagnosis of Ewing sarcoma, the pathologist must rule out multiple other differentials through the process of multiple immunohistochemical staining beyond the scope of this paper [27].

Concerning treatment, there is strong evidence that early and aggressive treatment leads to the most favorable outcomes $[8,14,17,23]$. In our case, there was strong evidence of a rapidly enlarging mass with rapidly progressive neurologic deficit. This warranted the first goal of operation to be decompression of the spinal cord with the proposed laminectomy. In other cases, if the mass was not producing rapidly progressive neurologic change, an argument may be made to first proceed with a needle biopsy and possible preoperative chemotherapy, shrinking the tumor and promoting an increased chance of successful gross total resection, followed 
by radiation therapy. Regardless, there is strong evidence that an aggressive multimodal treatment approach involving surgery, chemotherapy, and local radiation therapy increases the chance of a successful outcome $[8,17,23]$.

Survivorship of primary EES of the spine post treatment was fairly consistent across different studies. A study by Venkateswaran from St. Jude's Children's Research Hospital of 33 patients showed an estimated 5-year survival rate of $48.1 \%( \pm 8.9 \%)$ [28]. This was comparable to the Mayo Clinic study that showed a 5-year disease-free survival among its 125 patients at $45 \%$ for nonsacral lesions. Interestingly, this study also noted a $60 \% 5$-year disease-free survival rate for sacral lesions [5]. One world review of the literature performed by an Italian group found various 5 -year survival rates for primary spinal canal EES ranging from $0 \%$ to $37.5 \%[29,30]$.

\section{Conclusions}

The occurrence of an extraskeletal Ewing's sarcoma remains rare, which typically affects adolescents with a male preponderance. It is especially rare to be found in the spine, especially the cervical spine. We have shown with this case report and in a review of prior literature that it is not unheard of for EES to present in the spine, regardless of age, and in our case, even in late adulthood. While it remains rare for an EES to present in the spine, it should remain a viable differential diagnosis of an ill-defined spinal mass noted on imaging, independent of the patient's age. Standard treatment often involves a multidisciplinary approach of surgery, chemotherapy, and radiation therapy with the particular preferential order determined on a case-by-case basis and has a survivorship of approximately $45 \%$ at 5 years after treatment.

\section{Consent}

Written informed consent was obtained from the patient for publication of this case report and any accompanying images. A copy of the written consent is available for review.

\section{Conflict of Interests}

All authors of this paper declare no potential conflict of interests.

\section{Authors' Contribution}

Primary paper writing was performed by Marshall T. Holland and editing was performed by all authors. All authors have read and approved the final paper.

\section{References}

[1] N. Riggi and I. Stamenkovic, "The biology of Ewing sarcoma," Cancer Letters, vol. 254, no. 1, pp. 1-10, 2007.

[2] O. Javery, K. Krajewski, K. O’Regan et al., “A to Z of extraskeletal Ewing sarcoma family of tumors in adults: imaging features of primary disease, metastatic patterns, and treatment responses,"
The American Journal of Roentgenology, vol. 197, no. 6, pp. W1015-W1022, 2011.

[3] W. A. Kampman, J. M. Kros, T. H. R. de Jong, and M. H. Lequin, "Primitive neuroectodermal tumours (PNETs) located in the spinal canal; the relevance of classification as central or peripheral PNET: case report of a primary spinal PNET occurence with a critical literature review," Journal of Neuro-Oncology, vol. 77, no. 1, pp. 65-72, 2006.

[4] A. de Tommasi, C. de Tommasi, G. Occhiogrosso et al., "Primary intramedullary primitive neuroectodermal tumor (PNET)-case report and review of the literature," European Journal of Neurology, vol. 13, no. 3, pp. 240-243, 2006.

[5] H. Ilaslan, M. Sundaram, K. K. Unni, and M. B. Dekutoski, "Primary Ewing's sarcoma of the vertebral column," Skeletal Radiology, vol. 33, no. 9, pp. 506-513, 2004.

[6] M. R. Grubb, B. L. Currier, D. J. Pritchard, and M. J. Ebersold, "Primary Ewing's sarcoma of the spine," Spine, vol. 19, no. 3, pp. 309-313, 1994.

[7] G. Bozkurt, S. Ayhan, C. C. Turk, A. Akbay, F. Soylemezoglu, and S. Palaoglu, "Primary extraosseous Ewing sarcoma of the cervical epidural space. Case illustration," Journal of Neurosurgery: Spine, vol. 6, no. 2, article 192, 2007.

[8] J. G. Kennedy, S. Eustace, R. Caulfield, D. J. Fennelly, B. Hurson, and K. S. O'Rourke, "Extraskeletal Ewing's sarcoma: a case report and review of the literature," Spine, vol. 25, no. 15, pp. 1996-1999, 2000.

[9] S. Ali, K. MacKenzie, R. Reid, G. O’Neill, and I. Ganly, “Cervical extraskeletal Ewing's sarcoma: case report demonstrating radiological features and management," The Journal of Laryngology \& Otology, vol. 122, no. 9, pp. 998-1001, 2008.

[10] G. A. Cabral, C. F. Nunes, J. O. Melo Jr. et al., "Peripheral primitive neuroectodermal tumor of the cervical spine," Surgical Neurology International, vol. 3, article 91, 2012.

[11] D. P. Hao, W. J. Xu, Z. C. Wang et al., "Primary Ewing's sarcoma of the cervical vertebra: one case report and literature review," Journal of Pediatric Orthopedics Part B, vol. 19, no. 3, pp. 276880, 2010.

[12] G. Kara, "Spinal cord Ewing's sarcoma metastasis: presentation of one case," Annals of Nuclear Medicine, vol. 18, no. 7, pp. 623626, 2004.

[13] M. Kogawa, T. Asazuma, K. Iso et al., "Primary cervical spinal epidural Extra-osseous Ewing's sarcoma," Acta Neurochirurgica, vol. 146, no. 9, pp. 1051-1053, 2004.

[14] G. Martin Garcia, J. J. Cruz Hernandez, P. Sanchez et al., "Ewing's sarcoma in the second cervical vertebra," Annals of Oncology, vol. 2, no. 7, pp. 521-522, 1991.

[15] P. Mukhopadhyay, M. Gairola, M. C. Sharma, S. Thulkar, P. K. Julka, and G. K. Rath, "Primary spinal epidural extraosseous Ewing's sarcoma: report of five cases and literature review," Australasian Radiology, vol. 45, no. 3, pp. 372-379, 2001.

[16] E. Ozturk, H. Mutlu, G. Sonmez, F. V. Aker, C. C. Basekim, and E. Kizilkaya, "Spinal epidural extraskeletal Ewing sarcoma," Journal of Neuroradiology, vol. 34, no. 1, pp. 63-67, 2007.

[17] M. J. A. Sharafuddin, F. S. Haddad, P. W. Hitchon, S. F. Haddad, and G. Y. El-Khoury, "Treatment options in primary Ewing's sarcoma of the spine: report of seven cases and review of the literature," Neurosurgery, vol. 30, no. 4, pp. 610-619, 1992.

[18] J. H. Shin, H. K. Lee, S. C. Rhim, K. J. Cho, C. G. Choi, and D. C. Suh, "Spinal epidural extraskeletal Ewing sarcoma: MR findings in two cases," The American Journal of Neuroradiology, vol. 22, no. 4, pp. 795-798, 2001. 
[19] C. Villas and M. San Julian, "Ewing's tumor of the spine: report on seven cases including one with a 10-year follow-up," European Spine Journal, vol. 5, no. 6, pp. 412-417, 1996.

[20] F. Vincentelli, G. Caruso, and D. Figarella-Branger, "Primary intradural Ewing's sarcoma of the cauda equina presenting with acute bleeding," Acta Neurochirurgica, vol. 152, no. 3, pp. 563564, 2010.

[21] D. Gulati, A. N. Aggarwal, S. Kumar, and S. Chaturvedi, "Primary Ewing's sarcoma of the second cervical vertebra: a rare entity," Journal of Pediatric Orthopaedics Part B, vol. 20, no. 6, pp. 408-412, 2011.

[22] M. Tefft, G. F. Vawter, and A. Mitus, "Paravertebral 'round cell' tumors in children," Radiology, vol. 92, no. 7, pp. 1501-1509, 1969.

[23] C.-F. Xie, M.-Z. Liu, and M. Xi, "Extraskeletal Ewing's sarcoma: a report of 18 cases andliterature review," Chinese Journal of Cancer, vol. 29, no. 4, pp. 420-424, 2010.

[24] T. Yasuda, K. Suzuki, M. Kanamori et al., "Extraskeletal Ewing's sarcoma of the thoracic epidural space: case report and review of the literature," Oncology Reports, vol. 26, no. 3, pp. 711-715, 2011.

[25] C. V. Gopalakrishnan, A. Shrivastava, H. V. Easwer, and S. Nair, "Primary Ewing's sarcoma of the spine presenting as acute paraplegia," Journal of Pediatric Neurosciences, vol. 7, no. 1, pp. 64-66, 2012.

[26] S. Tsutsumi, Y. Yasumoto, A. Manabe, I. Ogino, H. Arai, and M. Ito, "Magnetic resonance imaging appearance of primary spinal extradural Ewing's sarcoma: case report and literature review," Clinical Neuroradiology, vol. 23, no. 2, pp. 81-85, 2013.

[27] B. W. Scheithauer and B. M. Egbert, "Ewing's sarcoma of the spinal epidural space: report of two cases," Journal of Neurology Neurosurgery and Psychiatry, vol. 41, no. 11, pp. 1031-1035, 1978.

[28] L. Venkateswaran, C. Rodriguez-Galindo, T. E. Merchant, C. A. Poquette, B. N. Rao, and A. S. Pappo, "Primary Ewing tumor of the vertebrae: clinical characteristics, prognostic factors, and outcome," Medical and Pediatric Oncology, vol. 37, no. 1, pp. 3035, 2001.

[29] G. Bacci, S. Boriani, A. Balladelli et al., "Treatment of nonmetastatic Ewing's sarcoma family tumors of the spine and sacrum: the experience from a single institution," European Spine Journal, vol. 18, no. 8, pp. 1091-1095, 2009.

[30] G.-J. J. L. Kaspers, W. Kamphorst, M. van de Graaff, A. M. van Alphen, and A. J. P. Veerman, "Primary spinal epidural extraosseous Ewing's sarcoma," Cancer, vol. 68, no. 3, pp. 648654, 1991.

[31] J. A. Ellis, R. J. Rothrock, G. Moise et al., "Primitive neuroectodermal tumors of the spine: a comprehensive review with illustrative clinical cases," Neurosurgical Focus, vol. 30, no. 1, p. E1, 2011. 


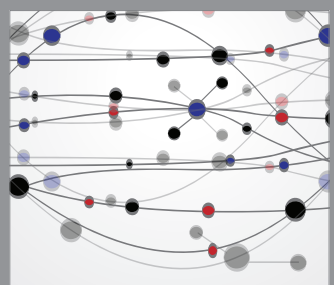

The Scientific World Journal
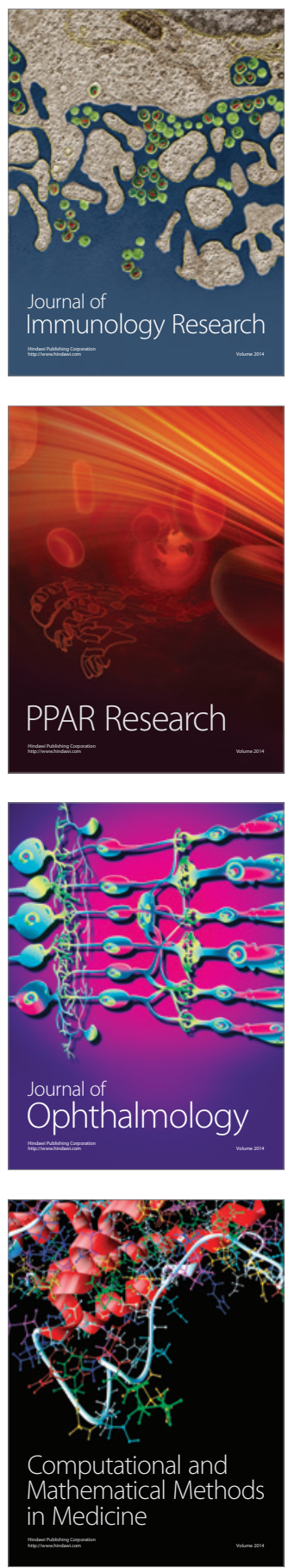

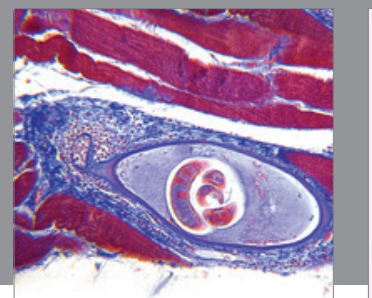

Gastroenterology

Research and Practice
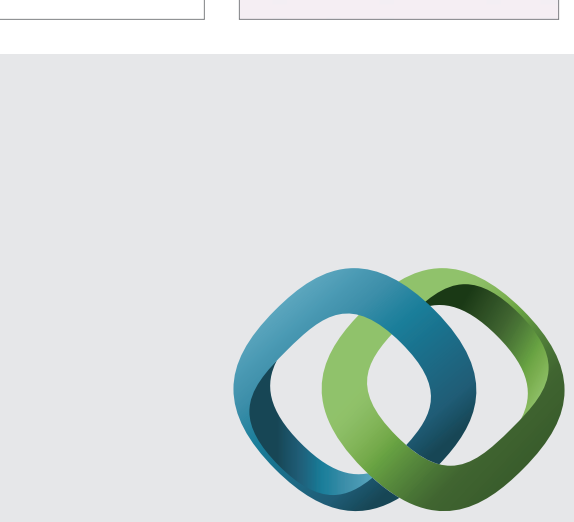

\section{Hindawi}

Submit your manuscripts at

http://www.hindawi.com
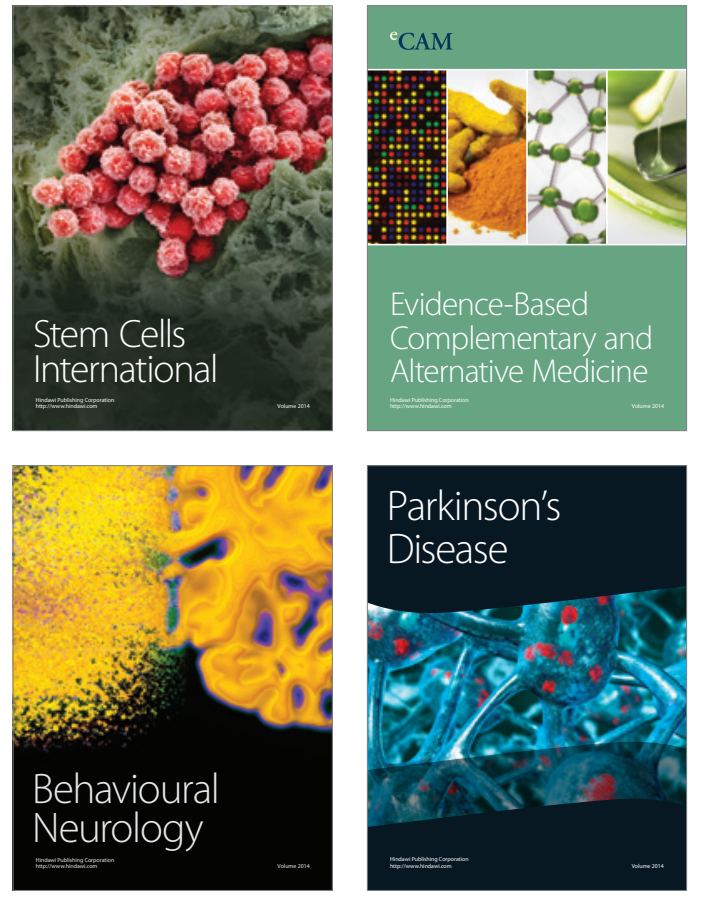
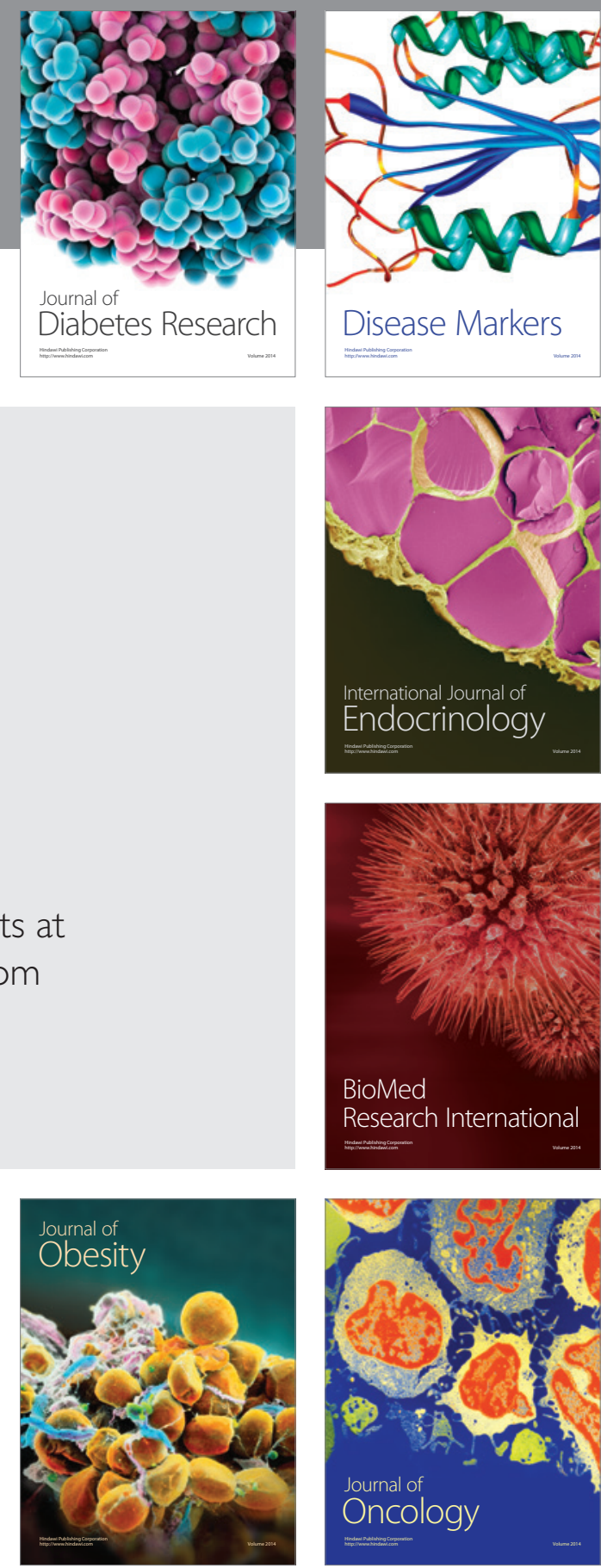

Disease Markers
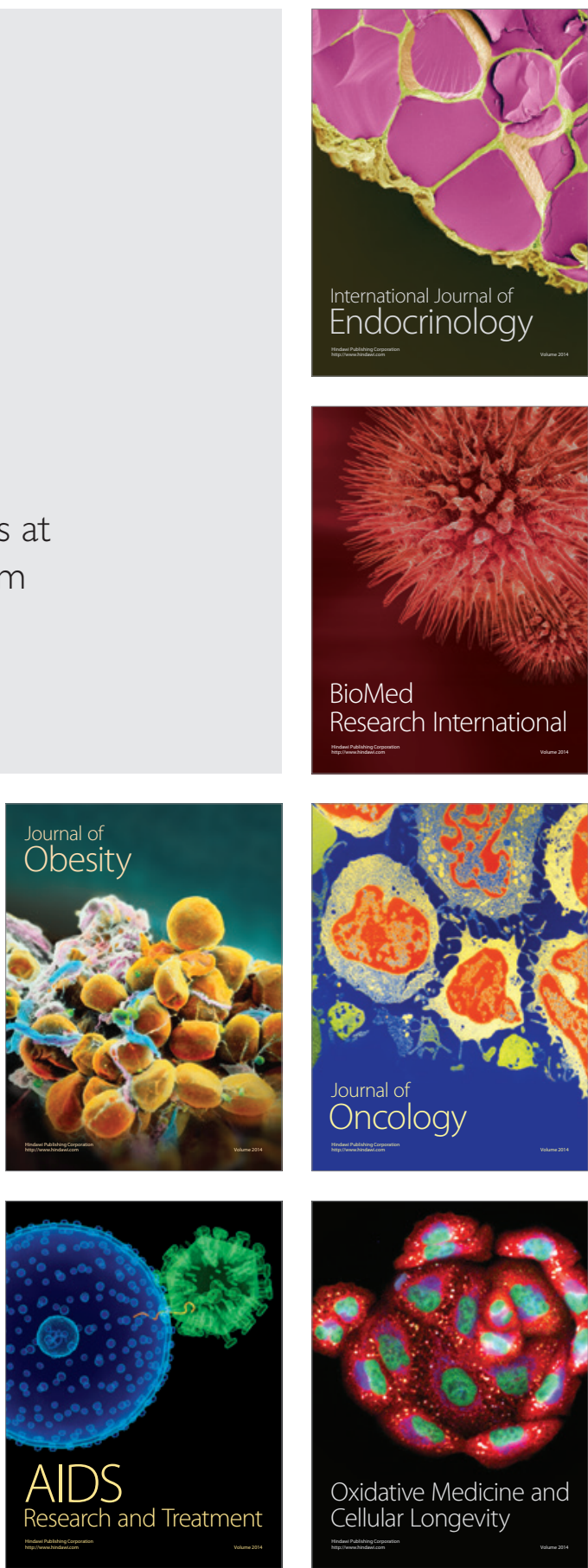